\title{
Predictive Design Model for Low-Dimensional Organic-Inorganic Halide Perovskites Assisted by Machine Learning
}

Ruiyang Lyu, Curtis E. Moore, Tianyu Liu, Yongze Yu, and Yiying $\mathrm{Wu}^{*}$

Machine Learning Part:

Software Libraries: Open-source code package such as Pandas and NumPy were used for data import and processing. Learning models in Scikit-learn were exported for data training, testing and model evaluation. All plots were drawn by Matplotlib. Jupyte Notebook 5.7.8 was used for code creation based on Python Language.

Feature Creation: JChem for Office (Excel) was used for chemical database access, feature selection, calculation, and reporting Different types of descriptor functions such as Charge, Element Analysis, Geometry, and Topological Analysis were used to calculate 40 features of the studied 86 ammoniums. In general, there are two types of functions. One is based on the whole molecule, such as the molecular polarizability, Weiner Index and Platt Index, etc. The other type focuses on a specific atom in the whole molecule, Nitrogen (N) in this paper, like Steric Effect Index, Atomic Polarizability and Eccentricity, etc. JChem for Office version: 19.9.0.467, 2019, ChemAxon (http://www.chemaxon.com)

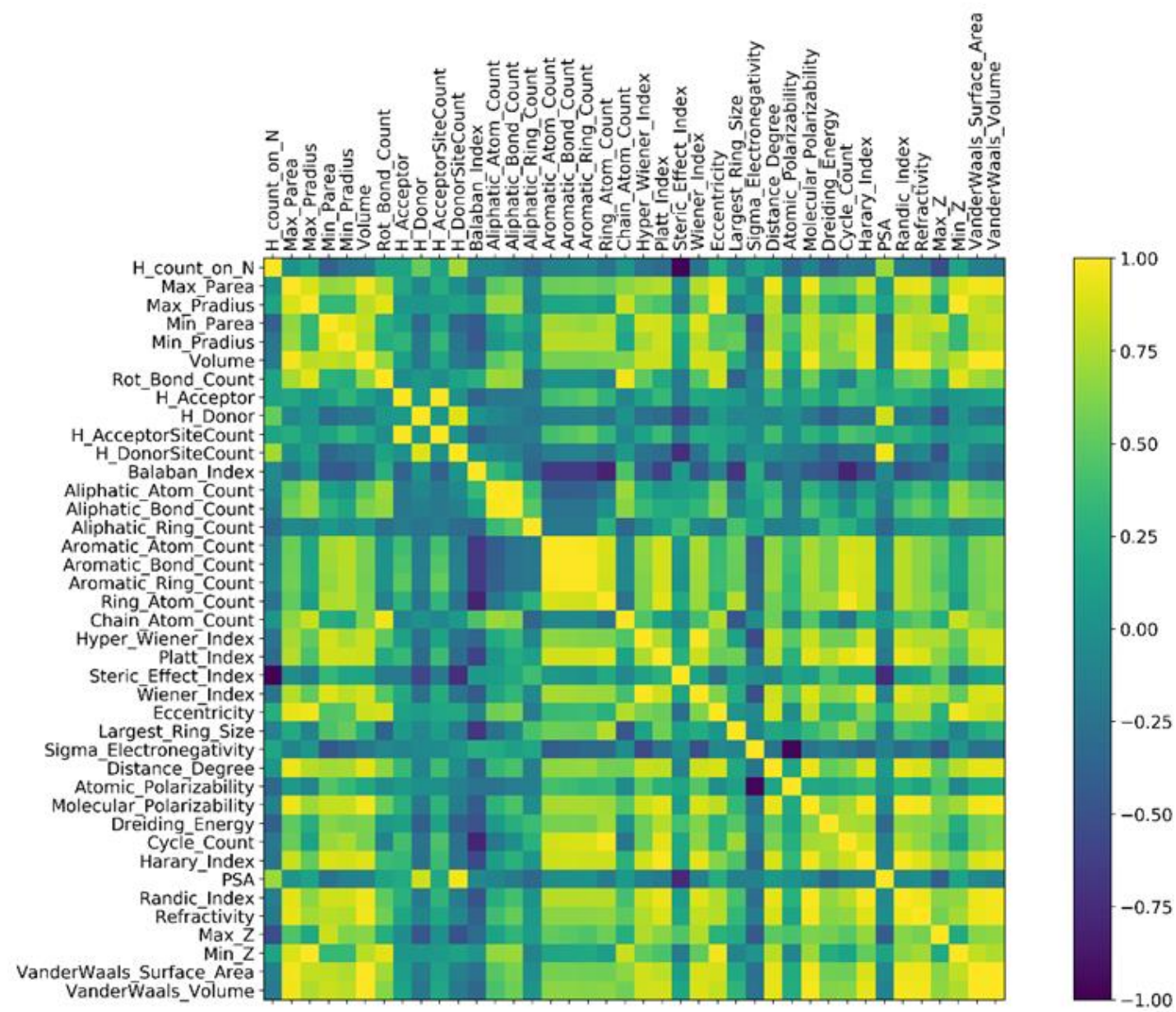

Figure S1. Correlation Matrix of 40 features. 
Feature Filtration: Selection of a wide range of features might introduce highly-correlated features. Therefore, feature filtering which can decrease the redundancy of the dataset is a necessary step. Pearson correlation coefficient (PCC) is widely used for feature elimination. PCC of any two features F1 and F2 is defined as:

$$
\rho_{F 1, F 2}=\frac{\operatorname{cov}\left(F_{1}, F_{2}\right)}{\sigma_{F 1} \sigma_{F 2}}
$$

Where $\operatorname{cov}(\mathrm{F} 1, \mathrm{~F} 2)$ is the covariance function which describes how the two features change together, $\sigma \mathrm{F} 1$ and $\sigma \mathrm{F} 2$ are the standard deviation of F1 and F2. Based on the mathematic expression, PCC should be a number between -1 and 1 . For these feature pairs with PCC close to -1 or 1 , they are highly negative or positive correlated with each other respectively. Therefore, high correlated features should be eliminated to prevent overfitting of the model. PCCs were calculated for each features pair and the correlation matrix is shown in Figure S1. A cut-off correlation score of 0.95 was set to group the features with high correlation and only one feature was left in each group. 21 features were removed after the filtering; and the PCCs matrix of the rest 21 features are shown in Figure S2.

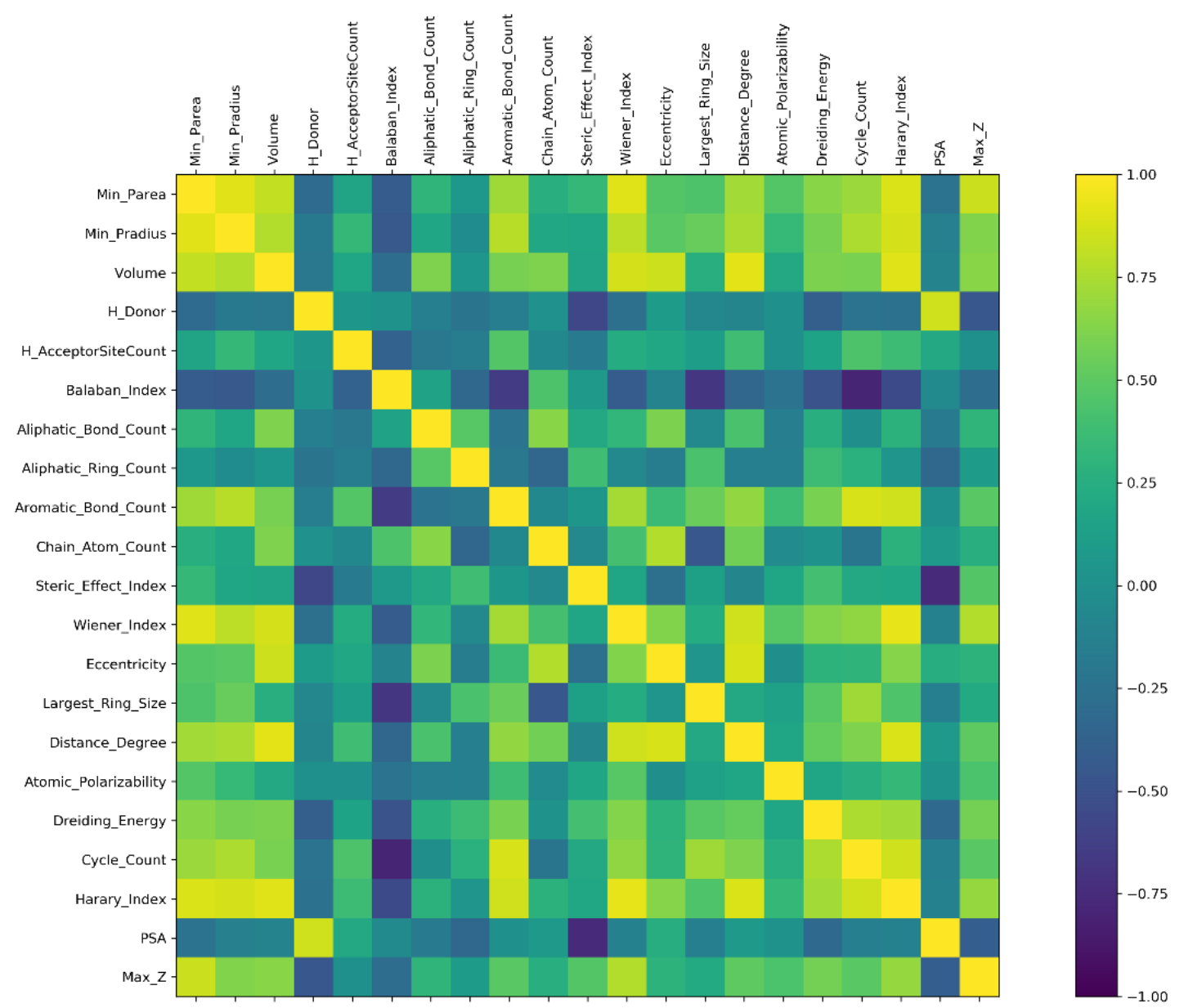

Figure S2. Correlation matrix of the selected 21 features

Table S1: Description of 40 unfiltered features

\begin{tabular}{|lcl|}
\hline Feature Name & $\begin{array}{c}\text { If function on an } \\
\text { atom }(\mathbf{Y} / \mathbf{N})\end{array}$ & Description \\
\hline Aliphatic_Atom_Count & $\mathbf{N}$ & Calculate the number of aliphatic atoms \\
\hline Aliphatic_Bond_Count & $\mathbf{N}$ & Calculate the number of aliphatic bonds \\
\hline Aliphatic_Ring_Count & $\mathbf{N}$ & Calculate the number of aliphatic rings \\
\hline Aromatic_Atom_Count & $\mathbf{N}$ & Calculate the number of aromatic atoms \\
\hline Aromatic_Bond_Count & $\mathbf{N}$ & Calculate the number of aromatic bonds \\
\hline Aromatic_Ring_Count & $\mathbf{N}$ & Calculate the number of aromatic rings \\
\hline
\end{tabular}




\begin{tabular}{|c|c|c|}
\hline Atomic_Polarizability & $\mathbf{Y}$ & Calculate atomic polarizability of a selected atom \\
\hline Balaban_Index & $\mathbf{N}$ & Calculate the Balaban Index (the average distance-sum connectivity) \\
\hline Chain_Atom_Count & $\mathbf{N}$ & Calculate the number of chain atoms \\
\hline Cycle_Count & $\mathbf{N}$ & Calculate the cyclomatic number \\
\hline Distance_Degree & $\mathbf{Y}$ & $\begin{array}{l}\text { Calculate the distance degree of a selected atom } \\
\text { (the sum of an entire row in the distance matrix) }\end{array}$ \\
\hline Dreiding_Energy & $\mathbf{N}$ & Calculate the dreiding energy of the molecule/ion \\
\hline Eccentricity & $\mathbf{Y}$ & $\begin{array}{l}\text { Calculate the eccentricity of a selected atom } \\
\text { (the maximal number of a row in the distance matrix) }\end{array}$ \\
\hline Harary_Index & $\mathbf{N}$ & Calculate the Harary Index \\
\hline H_AcceptorCount & $\mathbf{N}$ & Calculate the number of hydrogen bond acceptor atoms \\
\hline H_AcceptorSiteCount & $\mathbf{N}$ & $\begin{array}{l}\text { Calculate the multiplicity of hydrogen bond acceptor } \\
\text { (the sum of atomic multiplicity) }\end{array}$ \\
\hline H_count_on_N & $\mathbf{Y}$ & Calculate the number of hydrogens on nitrogen atom \\
\hline H_DonorCount & $\mathbf{N}$ & Calculate the number of hydrogen bond donor atoms \\
\hline H_DonorSiteCount & $\mathbf{N}$ & $\begin{array}{l}\text { Calculate the multiplicity of hydrogen bond donor } \\
\text { (the sum of atomic multiplicity) }\end{array}$ \\
\hline Hyper_Wiener_Index & $\mathbf{N}$ & Calculate the Hyper-Wiener index \\
\hline Largest_Ring_Size & $\mathbf{N}$ & Calculate the size of the largest ring (the number of atoms in the ring) \\
\hline Max_Projection_area & $\mathbf{N}$ & Calculate the maximal projection area of the molecule/ion \\
\hline Max_Projection_radius & $\mathbf{N}$ & Calculate the maximal projection radius of the molecule/ion \\
\hline MaxZ & $\mathbf{N}$ & Calculate the maximal $\mathrm{Z}$ coordinate in the bonding box of the molecule/ion \\
\hline Min_Projection_area & $\mathbf{N}$ & Calculate the minimal projection area of the molecule/ion \\
\hline Min_Projection_radius & $\mathbf{N}$ & Calculate the minimal projection radius of the molecule/ion \\
\hline MinZ & $\mathbf{N}$ & Calculate the minimal $\mathrm{Z}$ coordinate in the bonding box of the molecule/ion \\
\hline Molecular_Polarizability & $\mathbf{N}$ & Calculate the molecular polarizability of a selected atom \\
\hline platt_Index & $\mathbf{N}$ & Calculate the plat index (the sum of the edge degree) \\
\hline Polar_Suface_Area & $\mathbf{N}$ & Calculate the polar surface area \\
\hline Randic_Index & $\mathbf{N}$ & Calculate the randic index \\
\hline Refractivity & $\mathbf{N}$ & Calculate the molar refractivity \\
\hline Ring_Atom_Count & $\mathbf{N}$ & Calculate the number of ring atoms \\
\hline Rot_Bond_Count & $\mathbf{N}$ & Calculate the number of ring bonds \\
\hline Sigma_Electronegativity & $\mathbf{Y}$ & Calculate the atomic sigma orbital electronegativity \\
\hline Steric_Effect_Index & $\mathbf{Y}$ & Calculate the steric effect index of a selected atom \\
\hline Vander Waals_Surface_Area & $\mathbf{N}$ & Calculate the Vander Waals surface area of the molecule/ion \\
\hline Vander Waals_Volume & $\mathbf{N}$ & Calculate the Vander Waals volume of the molecule/ion \\
\hline Volume & $\mathbf{N}$ & Calculate the molecular volume \\
\hline Wiener_Index & $\mathbf{N}$ & Calculate the wiener index \\
\hline
\end{tabular}

Data Splitting: The feature dataset was split into training set and test set with the ratio of 80:20 in percentage. The model was trained based on the training set and then evaluated using the test set to determine its ability for unseen data prediction. For the accuracy of the model evaluation, the data was randomly split 200 times. The average scores were reported after model training.

\section{Machine learning algorithm in sckit-learning library for this work:}

$\operatorname{SVM}(\mathrm{rbf})$ :

$\operatorname{SVC}(\mathrm{C}=10$, cache_size $=200$, class_weight $=$ None, $\operatorname{coef} 0=0.0$,

decision_function_shape $=$ 'ovr', degree $=3$, gamma='auto_deprecated',

kernel='rbf', max_iter $=-1$, probability $=$ False, random_state $=$ None,

shrinking $=$ True, tol $=0.001$, verbose $=$ False)

Logistic Regression (11):

LogisticRegression $(C=1.0$, class_weight $=$ None, dual=False, fit_intercept $=$ True, 
intercept_scaling=1, max_iter=100, multi_class='warn',

$\mathrm{n} \_$jobs=None, penalty='l1', random_state $=$None, solver='warn',

tol $=0.0001$, verbose $=0$, warm_start $=$ False)

Logistic Regression (12):

LogisticRegression $(\mathrm{C}=1.0$, class_weight $=$ None, dual $=$ False, fit_intercept $=$ True,

intercept_scaling $=1$, max_iter $=100$, multi_class='warn',

n_jobs $=$ None, penalty='12', random_state $=$ None, solver='warn',

tol $=0.0001$, verbose $=0$, warm_start $=$ False)

\section{KNN:}

KNeighborsClassifier(algorithm='auto', leaf_size $=30$, metric='minkowski',

metric_params $=$ None, $n \_j o b s=$ None, $n \_$neighbors $=1, \mathrm{p}=2$,

weights='uniform')

Decision Tree:

DecisionTreeClassifier(class_weight $=$ None, criterion='gini', max_depth $=3$,

max_features=None, max_leaf_nodes=None,

min_impurity_decrease $=0.0$, min_impurity_split $=$ None,

min_samples_leaf $=1$, min_samples_split $=2$,

min_weight_fraction_leaf $=0.0$, presort $=$ False, random_state $=$ None,

splitter='best')

Gaussin Naïve Bayes:

GaussianNB(priors=None, var_smoothing $=1$ e-09)

Hyperparameter Tuning: Hyperparameter is important to prevent high variance or bias errors during the traning process. For the LR (11) mode that was chosen for this work, the hyperparameter tuning is shown in Figure S3. It is clear to see when C=1, the highest performance (highest test score with less difference from the traning score) can be obtained.

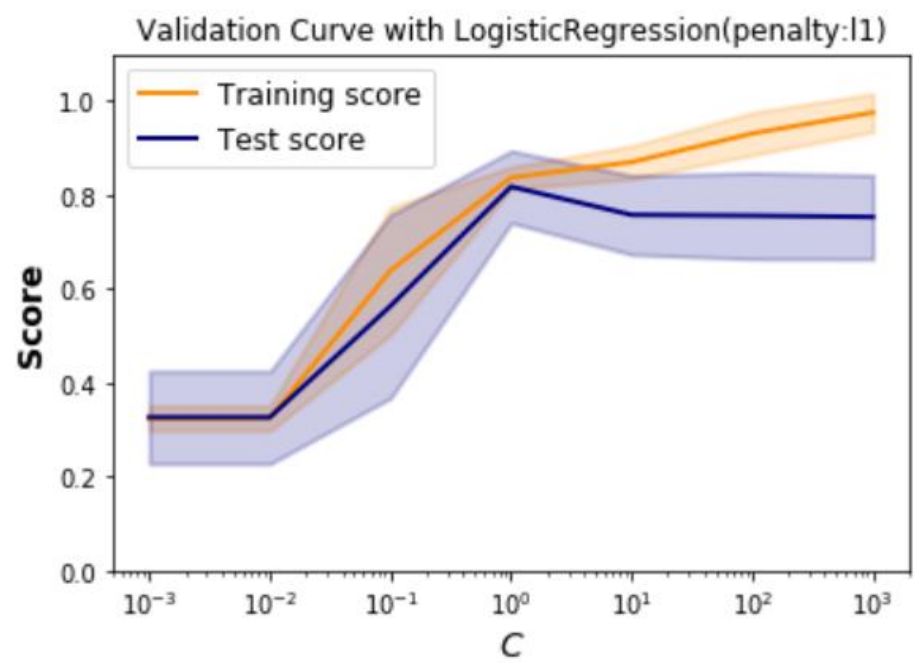

Figure S3. The hyperparameter tuning for LR (11) mode. 


\section{Calculation demonstration}

The Steric Effect Index (STEI) calculation for tetramethylammonium and cyclobutylammonium is shown below:

Tetramethylammonium<smiles>C[N+](C)(C)C</smiles>

\begin{tabular}{|l|l|l|l|l|l|}
\hline & $\mathrm{N}$ & $\mathrm{C} 1$ & $\mathrm{C} 2$ & $\mathrm{C} 3$ & $\mathrm{C} 4$ \\
\hline $\mathrm{N}$ & 0 & 1 & 1 & 1 & 1 \\
\hline $\mathrm{C} 1$ & 1 & 0 & 2 & 2 & 2 \\
\hline C2 & 1 & 2 & 0 & 2 & 2 \\
\hline C3 & 1 & 2 & 2 & 0 & 2 \\
\hline C4 & 1 & 2 & 2 & 2 & 0 \\
\hline
\end{tabular}

STEI $=\frac{1}{1^{3}} \times 4=4$

\section{Cyclobutylammonium}

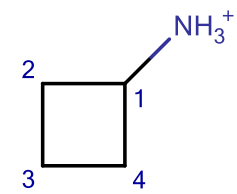

\begin{tabular}{|l|l|l|l|l|l|}
\hline & $\mathrm{N}$ & $\mathrm{C} 1$ & $\mathrm{C} 2$ & $\mathrm{C} 3$ & $\mathrm{C} 4$ \\
\hline $\mathrm{N}$ & 0 & 1 & 2 & 3 & 2 \\
\hline $\mathrm{C} 1$ & 1 & 0 & 1 & 2 & 1 \\
\hline $\mathrm{C} 2$ & 2 & 1 & 0 & 1 & 2 \\
\hline $\mathrm{C} 3$ & 3 & 2 & 1 & 0 & 1 \\
\hline $\mathrm{C} 4$ & 2 & 1 & 2 & 1 & 0 \\
\hline
\end{tabular}

$S T E I=\frac{1}{1^{3}}+\frac{1}{2^{3}}+\frac{1}{3^{3}}+\frac{1}{2^{3}}=1.287$

The Eccentricity $(\mathrm{Ec})$ calculation for cyclooctanammonium and octylammonium is shown below:

Cyclooctanammonium

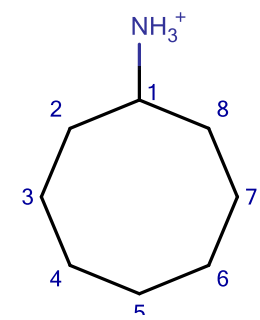

\begin{tabular}{|l|l|l|l|l|l|l|l|l|l|}
\hline & $\mathrm{N}$ & $\mathrm{C} 1$ & $\mathrm{C} 2$ & $\mathrm{C} 3$ & $\mathrm{C} 4$ & $\mathrm{C} 5$ & $\mathrm{C6}$ & $\mathrm{C7}$ & $\mathrm{C} 8$ \\
\hline $\mathrm{N}$ & 0 & 1 & 2 & 3 & 4 & 5 & 4 & 3 & 2 \\
\hline
\end{tabular}

$E c=5$

Octylammonium<smiles>CCCCCCCC[NH]</smiles>

\begin{tabular}{|l|l|l|l|l|l|l|l|l|l|}
\hline & $\mathrm{N}$ & $\mathrm{C} 1$ & $\mathrm{C} 2$ & $\mathrm{C} 3$ & $\mathrm{C} 4$ & $\mathrm{C} 5$ & $\mathrm{C} 6$ & $\mathrm{C} 7$ & $\mathrm{C} 8$ \\
\hline $\mathrm{N}$ & 0 & 1 & 2 & 3 & 4 & 5 & 6 & 7 & 8 \\
\hline
\end{tabular}

$E c=8$ 
Experimental section

Starting Materials: PbI2 (99.999\%), hydroiodic acid (57\% wt. in water, distilled, stabilized, 99.95\%), 4,7,10-trioxa-1,13tridecanediamine (97\%), 3-isopropoxypropylamine ( $>98 \%), \mathrm{N}, \mathrm{N}$-diisopropylamine $(>99.5 \%)$, isopropylamine $(>99.5 \%)$, piperidine (>99.5\%) and 2-ethyl-1-hexylamine (98\%) were purchased from Sigma-Aldrich; diisopropylethylamine ( $>99.0 \%)$ was purchased from TCI America. All these chemicals were used as received.

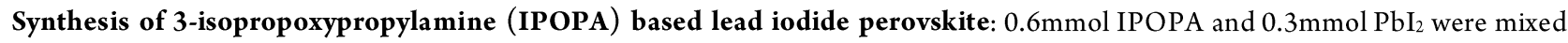
inside $0.5 \mathrm{~mL} 57 \% \mathrm{HI}$ solution. The yellow precipitate formed after mixing was collected by vacuum filtration and washed by ethyl acetate. For single crystal growth, diffusion method was applied. The yellow precipitate was re-dissolved into 57\% HI solution with diethyl ether as the anti-solvent. Yellow needle-like crystal was obtained after 1 day.

Synthesis of 2-ethyl-1-hexylamine (EHA) based lead iodide perovskite: $0.4 \mathrm{mmol} 2$-ethyl-1-hexylamine and $0.2 \mathrm{mmol} \mathrm{PbI} 2 \mathrm{were}$ dissolved inside $2 \mathrm{~mL} 57 \%$ HI solution. Orange precipitate formed immediately after mixing. The product was then collected by vacuum filtering and washed with dichloromethane. The single crystal was obtained by recrystallizing the precipitate inside ethyl acetate via evaporation.

Synthesis of piperidine based lead iodide perovskite: $0.4 \mathrm{mmol}$ piperidine and $0.2 \mathrm{mmol} \mathrm{PbI}_{2}$ were dissolved inside $57 \% \mathrm{HI}$ solution with a molar ration of 2:1. The yellow precipitate formed after mixing was collected by vacuum filtration and washed by ethyl acetate. For single crystal growth, the yellow precipitate was re-dissolved into $57 \% \mathrm{HI}$ solution. Diffusion method was used with diethyl ether as the anti-solvent. Yellow needle-like crystal was obtained after 1 2 days.

Synthesis of diisopropylamine (DIPA) based lead iodide perovskite: DIPA was protonated with equal molar HI by directly adding DIPA into $57 \% \mathrm{HI}$ solution. The white precipitate (DIPAHI) was collected by vacuum filtration and dried under $60^{\circ} \mathrm{C}$ inside vacuum oven. $0.4 \mathrm{mmol}$ DIPAHI and $0.2 \mathrm{mmol} \mathrm{PbI}_{2}$ were dissolved inside dimethylformamide. Dichloromethane was used as antisolvent for the single crystal growth. Yellow stick-like crystal was obtained after 1 2 days.

Synthesis of diisopropylethyla mine (DIPEA) based lead iodide perovskite: $0.6 \mathrm{mmol}$ DIPEA and $0.3 \mathrm{mmol}^{\mathrm{PbI}_{2}} \mathrm{were}$ dissolved inside $0.5 \mathrm{~mL} 57 \% \mathrm{HI}$ solution. The as-formed milky solution was heated up to $90^{\circ} \mathrm{C}$ with stirring and then gradually cooled down to room temperature. Yellow needle-like single crystals were formed during the cooling process.

Characterization: The powder X-ray diffraction was measured by a Bruker D8 Advance X-ray diffractometer with Cu Ka source and operated at $40 \mathrm{kV}, 40 \mathrm{~mA}$. The single crystal X-ray diffraction test was done by a Nonius Kappa diffractometer equipped with a Bruker APEX-II CCD and Mo Ka radiation $(\lambda=0.71073 \AA$ ). Data were integrated using the Bruker SAINT software program and scaled using the SADABS software program. Solution by direct methods (SHELXT) produced a complete phasing model for refinement. All nonhydrogen atoms were refined anisotropically by full-matrix least-squares (SHELXL-2014). All hydrogen atoms were placed using a riding model. Their positions were constrained relative to their parent atom using the appropriate HFIX command in SHELXL-2014. 


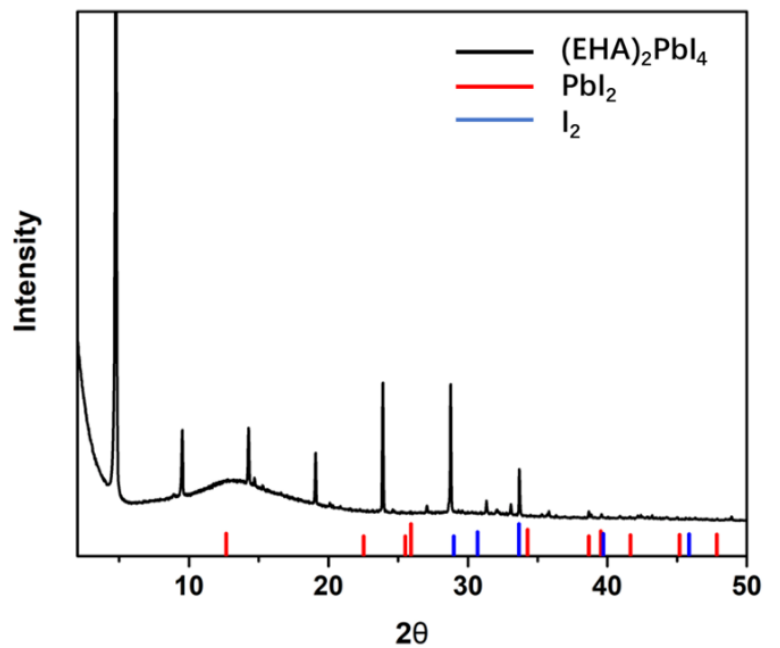

Figure S4. Powder XRD pattern of (EHA) $)_{2} \mathrm{PbI}_{4}$

Calculation of the layer distance based on the first peak position $\left(2 \theta=4.75^{\circ}\right)$ using Bragg's Law:

$$
d=\frac{\lambda}{2 \sin \theta}=\frac{1.544 \mathrm{~nm}}{2 \sin \left(\frac{4.75^{\circ}}{2}\right)}=1.863 \mathrm{~nm}
$$

Table S2. Unit cell information of the 5 new perovskites from the untested amines.

\begin{tabular}{|c|c|c|c|c|c|}
\hline Compound & (IPOPA)PbI ${ }_{3}$ & $(\mathrm{EHA})_{2} \mathrm{PbI}_{4}$ & $(\mathrm{DIPA}) \mathrm{PbI}_{3} \bullet \mathrm{DMF}$ & $(\mathbf{P P D}) \mathrm{PbI}_{3}$ & (DIPEA) $\mathrm{PbI}_{3}$ \\
\hline Formula & $\left(\mathrm{C}_{6} \mathrm{H}_{16} \mathrm{NO}\right) \mathrm{PbI}_{3}$ & $\left(\mathrm{C}_{8} \mathrm{H}_{20} \mathrm{~N}\right)_{2} \mathrm{PbI}_{4}$ & $\begin{array}{l}\left(\mathrm{C}_{6} \mathrm{H}_{16} \mathrm{~N}\right) \mathrm{PbI}_{3} \bullet \\
\mathrm{C}_{3} \mathrm{H}_{7} \mathrm{NO}\end{array}$ & $\left(\mathrm{C}_{5} \mathrm{H}_{12} \mathrm{~N}\right) \mathrm{PbI}_{3}$ & $\left(\mathrm{C}_{8} \mathrm{H}_{20} \mathrm{~N}\right) \mathrm{PbI}_{3}$ \\
\hline Crystal System & Monoclinic & Monoclinic & Triclinic & Orthorhombic & Orthorhombic \\
\hline Space group & $\mathrm{P} 2{ }_{1} / \mathrm{c}$ & $\mathrm{P} 2_{1} / \mathrm{c}$ & P-1 & $\mathrm{Cmcm}$ & $\mathrm{Pna}_{1}$ \\
\hline Unit cell & $a=12.476 \AA$ & $a=18.682 \AA$ & $\mathrm{a}=8.106 \AA$ & $a=8.707 \AA$ & $a=18.647 \AA$ \\
\hline \multirow[t]{5}{*}{ dimensions } & $\mathrm{b}=15.265 \AA$ & $\mathrm{b}=8.840 \AA$ & $\mathrm{b}=10.426 \AA$ & $\mathrm{b}=18.423 \AA$ & $b=20.509 \AA$ \\
\hline & $c=7.924 \AA$ & $\mathrm{c}=8.834 \AA$ & $c=11.474 \AA$ & $c=8.130 \AA$ & $c=8.353 \AA$ \\
\hline & $\alpha=90.000^{\circ}$ & $\alpha=90.000^{\circ}$ & $\alpha=92.895^{\circ}$ & $\alpha=90.000^{\circ}$ & $\alpha=90.000^{\circ}$ \\
\hline & $\beta=100.929^{\circ}$ & $\beta=95.370^{\circ}$ & $\beta=93.280^{\circ}$ & $\beta=90.000^{\circ}$ & $\beta=90.000^{\circ}$ \\
\hline & $\gamma=90.000^{\circ}$ & $\gamma=90.000^{\circ}$ & $\gamma=104.377^{\circ}$ & $\gamma=90.000^{\circ}$ & $\gamma=90.000^{\circ}$ \\
\hline Volume & $1481.8 \AA^{3}$ & $1452.6 \AA^{3}$ & $935.7 \AA^{3}$ & $1304.0 \AA^{3}$ & $3194.5 \AA^{3}$ \\
\hline $\mathrm{Z}$ & 4 & 2 & 2 & 4 & 8 \\
\hline Density & $3.165 \mathrm{~g} / \mathrm{cm}^{3}$ & $2.230 \mathrm{~g} / \mathrm{cm}^{3}$ & $2.709 \mathrm{~g} / \mathrm{cm}^{3}$ & $3.433 \mathrm{~g} / \mathrm{cm}^{3}$ & $2.986 \mathrm{~g} / \mathrm{cm}^{3}$ \\
\hline $\begin{array}{l}\text { Goodness-of-fit on } \\
\text { F2 }\end{array}$ & 1.118 & 1.044 & 1.075 & 1.070 & 1.045 \\
\hline $\begin{array}{l}\text { Final } R \text { indices } \\
{[I>2 \sigma(I)]}\end{array}$ & $\begin{array}{l}\mathrm{R} 1=0.0200 \\
\mathrm{wR} 2=0.0457\end{array}$ & $\begin{array}{l}\mathrm{R} 1=0.0243 \\
\mathrm{wR} 2=0.0594\end{array}$ & $\begin{array}{l}\mathrm{R} 1=0.0187 \\
\mathrm{wR} 2=0.0456\end{array}$ & $\begin{array}{l}\mathrm{R} 1=0.0193 \\
\mathrm{wR} 2=0.0435\end{array}$ & $\begin{array}{l}\mathrm{R} 1=0.0265 \\
\mathrm{wR} 2=0.0517\end{array}$ \\
\hline
\end{tabular}




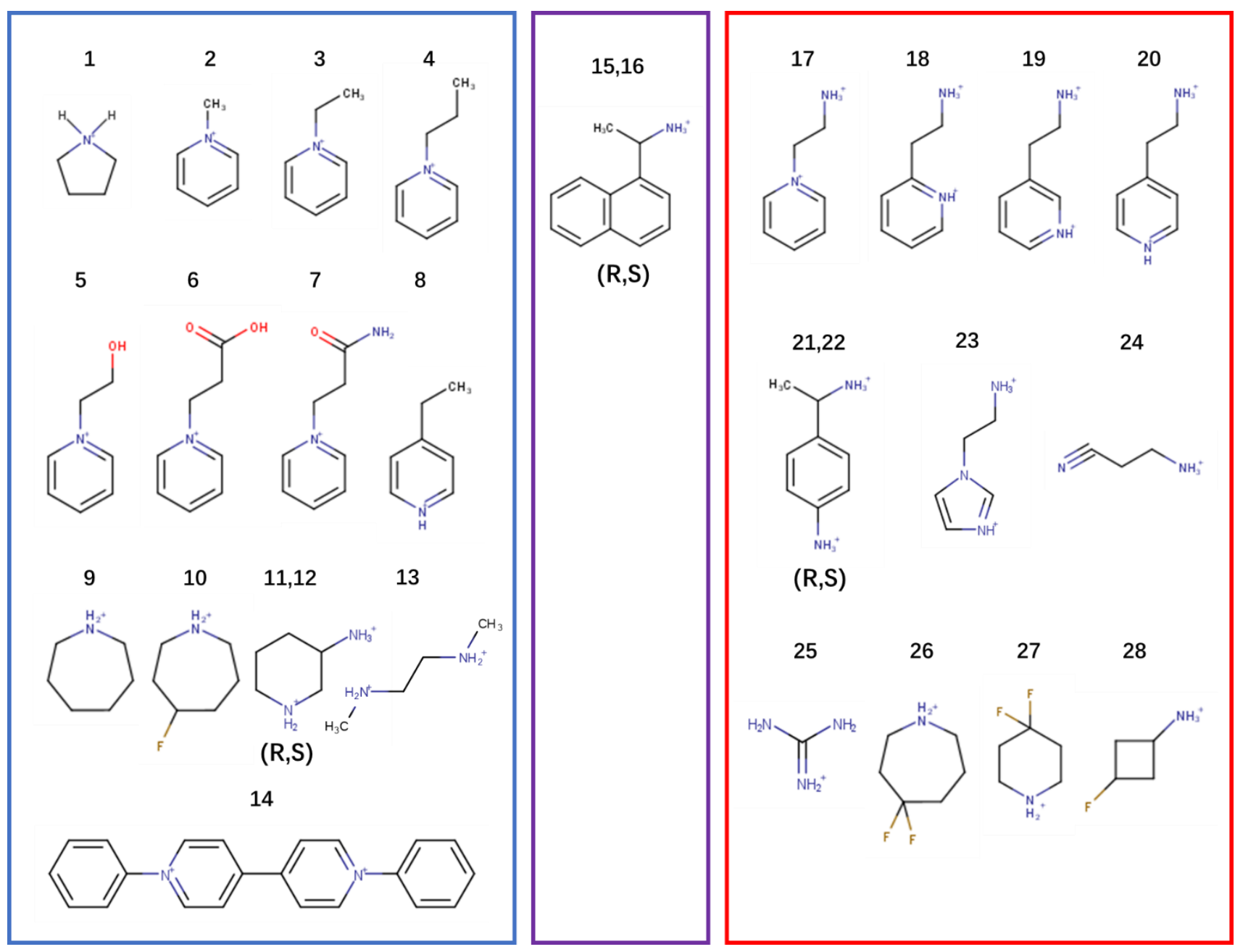

Figure S5. Summary of the 29 ammoniums: 1. pyrrolidinium ${ }^{1}$, 2. 1-methylpyridinium ${ }^{2}$, 3. 1-ethylpyridinium ${ }^{2}$, 4. 1-propylpyridinium ${ }^{2}, 5$. 1-(2-hydroxyethyl)pyridinium ${ }^{2}$, 6. 1-(2-carboxyethyl)pyridinium ${ }^{2}$, 7. 1-(2-carbamoylethyl)pyridinium², 8. 4-ethylpyridinium ${ }^{3}, 9$. azepanium $^{4}$, 10. 4-fluoroazepanium ${ }^{4}$, 11. 3R-3-azaniumylpiperidinium, 12. 3S-3-azaniumylpiperidinium, $13 . \quad \mathrm{N}, \quad \mathrm{N}^{\prime}-$ dimethylethylenediammonium $^{5}$, 14. phenyl viologen ${ }^{6}$, 15. (1R)-1-(naphthalen-1-yl)ethanammonium ${ }^{7}$, 16. (1S)-1-(naphthalen-1yl)ethanammonium ${ }^{7}$, 17. 1-(2-azaniumylethyl)pyridinium ${ }^{2}$, 18. 2-(2-azaniumylethyl)pyridinium², 19. 3-(2-azaniumylethyl) pyridinium $^{2}$, 20. 4-(2-azaniumylethyl)pyridinium ${ }^{2}, \quad 21 . \quad 4-[(1 \mathrm{R})-1$-azaniumylethyl $]$ anilinium $^{8}, \quad 22.4$-[(1S)-1-azaniumylethyl $]$ anilinium $^{8}, \quad 23$. Imidazolium ethyl ammonium ${ }^{9}$, 24. 3-Aminopropionitrile ${ }^{10}$, 25. Guanidinium ${ }^{11}$, 26. 4,4-difluoroazepanium ${ }^{4}$, $27 . \quad 4,4-$ difluoropiperidinium ${ }^{8}$, 28. 3-fluorocyclobutylammonium ${ }^{12}$.

Table S3. The test results of 28 ammoniums from recent publications.

\begin{tabular}{|c|c|c|c|}
\hline Ammonium Number & Log Odds of $\mathbf{P}_{\mathbf{2}}$ & Predicted Label & Reported Dimensionality \\
\hline 1 & - & Non-2D & $1 \mathrm{D}$ \\
\hline 2 & - & Non-2D & $1 \mathrm{D}$ \\
\hline 3 & - & Non-2D & $1 \mathrm{D}$ \\
\hline 4 & - & Non-2D & $1 \mathrm{D}$ \\
\hline 5 & - & Non-2D & $1 \mathrm{D}$ \\
\hline 6 & - & Non-2D & $1 \mathrm{D}$ \\
\hline 7 & - & Non-2D & $1 \mathrm{D}$ \\
\hline 8 & - & Non-2D & $1 \mathrm{D}$ \\
\hline 9 & - & Non-2D & \\
\hline
\end{tabular}




\begin{tabular}{|c|c|c|c|}
\hline 10 & - & Non-2D & $1 \mathrm{D}$ \\
\hline 11 & + & $2 \mathrm{D}$ & $1 \mathrm{D}$ \\
\hline 12 & + & $2 \mathrm{D}$ & $1 \mathrm{D}$ \\
\hline 13 & + & $2 \mathrm{D}$ & $2 \mathrm{D}$ \\
\hline 14 & - & $2 \mathrm{D}$ & $2 \mathrm{D}$ \\
\hline 15 & + & $2 \mathrm{D}$ & $2 \mathrm{D}$ \\
\hline 16 & - & Non-2D & $2 \mathrm{D}$ \\
\hline 17 & + & $2 \mathrm{D}$ & $2 \mathrm{D}$ \\
\hline 18 & + & $2 \mathrm{D}$ & $2 \mathrm{D}$ \\
\hline 19 & + & $2 \mathrm{D}$ & $2 \mathrm{D}$ \\
\hline 20 & + & $2 \mathrm{D}$ & $2 \mathrm{D}$ \\
\hline 21 & + & $2 \mathrm{D}$ & $2 \mathrm{D}$ \\
\hline 22 & + & $2 \mathrm{D}$ & $2 \mathrm{D}$ \\
\hline 23 & + & $2 \mathrm{D}$ & $2 \mathrm{D}$ \\
\hline 24 & + & $2 \mathrm{D}$ & $2 \mathrm{D}$ \\
\hline 25 & - & Non-2D & $2 \mathrm{D}$ \\
\hline 26 & + & Non-2D & $2 \mathrm{D}$ \\
\hline 28 & & + & \\
\hline
\end{tabular}

\section{References}

(1) Pham, N. D.; Yang, Y.; Hoang, M. T.; Wang, T.; Tiong, V. T.; Wilson, G. J.; Wang, H. 1D Pyrrolidinium Lead Iodide for Ef Fi Cient and Stable Perovskite Solar Cells. Energy Technol. 2020, 8, 1900918.

https://doi.org/10.1002/ente.201900918.

(2) Febriansyah, B.; Lekina, Y.; Ghosh, B. Molecular Engineering of Pure 2D Lead-Iodide Perovskite Solar Absorbers Displaying Reduced Band Gaps and Dielectric Confinement. ChemSusChem 2020, 2693-2701.

https://doi.org/10.1002/cssc.202000028.

(3) Duong, T. H.; Nobusue, S.; Tada, H. Preparation of Perovskite-Derived One Dimensional Single Crystals Based on EdgeShared Octahedrons with Pyridine Derivatives. J. Cryst. Growth 2020, 537 (December 2019), 125577.

https://doi.org/10.1016/j.jcrysgro.2020.125577.

(4) Chen, X.; Song, X.; Zhang, Z.; Zhang, H.; Pan, Q.; Yao, J.; You, Y.; Xiong, R. Confinement-Driven Ferroelectricity in a Two-Dimensional Hybrid Lead Iodide Perovskite. J. Am. Chem. Soc. 2020, 142 (22), 102 12-10218. https://doi.org/10.1021/jacs.0c03710.

(5) Ercan, E.; Cheng, X.; Ma, J.; Zhou, Y.; Fang, C.; Wang, J.; Li, J.; Wen, X.; Li, D. Optical Anisotropy of One-Dimensional Perovskite C 4 N 2 H 14 PbI 4 Crystals. J. Phys. Photonics 2020, 2, 14008. https://doi.org/10.1088/2515-7647/ab6743.

(6) Blundo, E.; Polimeni, A.; Meggiolaro, D.; Annibale, A. D.; Romagnoli, L.; Felici, M.; Latini, A. Brightly Luminescent and Moisture Tolerant Phenyl Viologen Lead Iodide Perovskites for Light Emission Applications. J. Phys. Chem. Lett. 2021, 12, 5456-5462. https://doi.org/10.1021/acs.jpclett.1c01271.

(7) Ishii, A.; Miyasaka, T. Direct Detection of Circular Polarized Light in Helical 1D Perovskite-Based Photodiode. Sci. Adv. 2020, 6 (46). https://doi.org/10.1126/SCIADV.ABD3274.

(8) Kim, D.; Dryzhakov, B.; Liu, Y.; Ovchinnikova, O. S.; Hu, B.; Kalinin, S. V.; Ahmadi, M. Ferroelectric and Charge Transport Properties in Strain-Engineered Two-Dimensional Lead Iodide Perovskites. Chem. Mater. 2021, 33 (11), 4077-4088. https://doi.org/10.1021/acs.chemmater.1c00679.

(9) Febriansyah, B.; Koh, T. M.; Lekina, Y.; Jamaludin, N. F.; Bruno, A.; Ganguly, R.; Shen, Z. X.; Mhaisalkar, S. G.; England, J. Improved Photovoltaic Efficiency and Amplified Photocurrent Generation in Mesoporous n = 1 Two-Dimensional 
Lead-Iodide Perovskite Solar Cells. Chem. Mater. 2019, 31 (3), 890-898.

https://doi.org/10.1021/acs.chemmater.8b04064.

(10) Li, Y.; Zhao, Y.; Cheng, H.; Zhao, K.; Wang, Z. S. Highly Efficient and Stable Pure Two-Dimensional Perovskite-Based Solar Cells with the 3-Aminopropionitrile Organic Cation. ACS Appl. Mater. Interfaces 2020, 12 (16), 18590-18595. https://doi.org/10.1021/acsami.0c02295.

(11) Nguyen-Tran, T.; Dinh, V. A.; Van Ly, N.; Luong, H. D.; Pham, D. T.; Truong, T. T.; Nguyen, H. Q.; Dao, Q. D.; Kim Tran, C. T.; Thu Bui, H. T.; Nguyen, D. T.; Ha Dang, M. N.; Thi Phan, V. V.; Truong, Q. D. Novel (110) DoubleLayered Guanidinium-Lead Iodide Perovskite Material: Crystal Structure, Electronic Structure, and Broad Luminescence. J. Phys. Chem. C 2021, 125 (1), 964-972. https://doi.org/10.1021/acs.jpcc.0c08540.

(12) Huang, C. R.; Li, P. F.; Luo, X. Z. A Two-Dimensional Organic-Inorganic Lead Iodide Perovskite: Poly[Bis(3Fluorocyclobutylammonium) [Di- $\mu$-Iodido-Diiodidoplumbate(VI)]]. Acta Crystallogr. Sect. C Struct. Chem. 2020, 76 (Vi), 1096-1099. https://doi.org/10.1107/S2053229620015272. 